\title{
The relationship between red blood cell deformability metrics and perfusion of an artificial microvascular network
}

\author{
Jose M. Sosa ${ }^{\mathrm{a}}$, Nathan D. Nielsen ${ }^{\mathrm{b}}$, Seth M. Vignes ${ }^{\mathrm{a}}$, Tanya G. Chen ${ }^{\mathrm{a}}$ \\ and Sergey S. Shevkoplyas ${ }^{\text {a,* }}$ \\ ${ }^{a}$ Department of Biomedical Engineering, Tulane University, New Orleans, LA, USA \\ ${ }^{\mathrm{b}}$ Department of Pulmonary Disease, Critical Care \& Environmental Medicine, Tulane University School \\ of Medicine, New Orleans, LA, USA
}

\begin{abstract}
The ability of red blood cells (RBC) to undergo a wide range of deformations while traversing the microvasculature is crucial for adequate perfusion. Interpretation of RBC deformability measurements performed in vitro in the context of microvascular perfusion has been notoriously difficult. This study compares the measurements of RBC deformability performed using micropore filtration and ektacytometry with the RBC ability to perfuse an artificial microvascular network (AMVN). Human RBCs were collected from healthy consenting volunteers, leukoreduced, washed and exposed to graded concentrations $(0-0.08 \%)$ of glutaraldehyde (a non-specific protein cross-linker) and diamide (a spectrin-specific protein cross-linker) to impair the deformability of RBCs. Samples comprising cells with two different levels of deformability were created by adding non-deformable RBCs (hardened by exposure to $0.08 \%$ glutaraldehyde) to the sample of normal healthy RBCs. Ektacytometry indicated a nearly linear decline in RBC deformability with increasing glutaraldehyde concentration. Micropore filtration showed a significant reduction only for concentrations of glutaraldehyde higher than $0.04 \%$. Neither micropore filtration nor ektacytometry measurements could accurately predict the AMVN perfusion. Treatment with diamide reduced RBC deformability as indicated by ektacytometry, but had no significant effect on either micropore filtration or the AMVN perfusion. Both micropore filtration and ektacytometry showed a linear decline in effective RBC deformability with increasing fraction of non-deformable RBCs in the sample. The corresponding decline in the AMVN perfusion plateaued above $50 \%$, reflecting the innate ability of blood flow in the microvasculature to bypass occluded capillaries. Our results suggest that in vitro measurements of RBC deformability performed using either micropore filtration or ektacytometry may not represent the ability of same RBCs to perfuse microvascular networks. Further development of biomimetic tools for measuring RBC deformability (e.g. the AMVN) could enable a more functionally relevant testing of RBC mechanical properties.
\end{abstract}

Keywords: Red blood cell deformability, micropore filtration, ektacytometry, artificial microvascular network, microfluidics

\section{Introduction}

The continual flow of blood through the human microvasculature ensures the transport of dissolved gases, nutrients and regulatory molecules throughout the body as well as the prompt removal of metabolic waste products. Adequate perfusion of the microvasculature depends on several important

\footnotetext{
${ }^{*}$ Corresponding author: Sergey S. Shevkoplyas, PhD, Department of Biomedical Engineering, Tulane University, 500 Lindy Boggs Building, New Orleans, LA 70118, USA. Tel.: +1 504314 2940; Fax: +1 504862 8779; E-mail: shevkop@ tulane.edu. 
hemorheological parameters including blood plasma viscosity, hematocrit, red blood cell (RBC) aggregation, and particularly the ability of individual RBCs to deform (e.g. contort, twist, change shape) when flowing through microvessels ranging in size from $100 \mu \mathrm{m}$ arterioles down to the smallest $3 \mu \mathrm{m}$ capillaries $[2,34,43,64,65,67,69]$. Alterations in RBC deformability have been associated with pathophysiological insults in conditions as diverse as diabetes mellitus, sickle cell anemia, malaria, sepsis, and postischaemic reperfusion [17, 19, 35, 45, 47, 62, 63]. A reduction in RBC deformability sometimes precedes more severe and often irreversible pathological changes in other vital organs and organ systems, and in some cases may even be the root cause of organ injury $[3,12,16,20,50,52,57]$.

A continuous research effort has been focused over the years on the development of instruments for measuring the mechanical response of RBCs to various deforming forces at either the single-cell or multicell level, and thus quantifying RBC "deformability" [46]. The two techniques most frequently utilized in the vast majority of research performed to date in this area (and perhaps most accessible in the clinical settings) are the micro-pore filtration assay $[9,21,25,39,40,53,55,56]$ and ektacytometry $[4,8,14,18$, 28-31, 37, 51, 61, 66, 71]. In this paper, we directly compare the measurements of RBC deformability performed using these two methodologies with the ability of RBCs to perfuse an artificial microvascular network (AMVN), a microfluidic device developed in our laboratory for modeling the dynamics of blood flow and traffic of circulating cells in the microvasculature $[13,23,59,60]$. We completed the comparison using RBC samples with cell deformability artificially impaired via graded exposure to glutaraldehyde (a non-specific protein cross-linker) and to diamide (a spectrin-specific cross-linker), both of which are frequently used to determine the sensitivity of various deformability metrics [1, 15, 24, 26, 61]. We found that the two methodologies were often in disagreement with each other, and that neither micro-pore filtration nor ektacytometry could accurately predict the ability of RBC samples to perfuse the AMVN. Our results support the notion that RBC deformability is not a unique property but is rather operationally defined by the measurement methodology, and emphasize the need for the development of biomimetic tools for a more relevant assessment of RBC mechanical properties.

\section{Materials and methods}

\subsection{Blood samples}

Human whole blood was collected from healthy consenting volunteers by venipuncture into $6 \mathrm{~mL}$ Vacutainer tubes $\left(\mathrm{K}_{2}\right.$ EDTA, BD, Franklin Lakes, NJ, USA). Plasma was removed by centrifugation $\left(800 \times \mathrm{g}\right.$ for 5 minutes, $\left.22^{\circ} \mathrm{C}\right)$ and discarded. Pelleted RBCs were re-suspended in $50 \mathrm{~mL}$ of phosphate buffered saline (PBS, Sigma, St. Louis, USA) and passed through a leukoreduction filter (Purcell NEO, Pall Corporation, Port Washington, NY, USA). The leukoreduced RBC suspension was washed in PBS once $\left(800 \times \mathrm{g}\right.$ for 5 minutes, $\left.22^{\circ} \mathrm{C}\right)$, and adjusted to a $40 \%$ hematocrit.

\subsection{Glutaraldehyde treatment}

The solution of glutaraldehyde ( $8 \%$ w/v, Sigma, St. Louis, USA) was diluted in PBS to form stock solutions with concentrations of glutaraldehyde corresponding to twice the targeted final concentrations. RBC samples (leukoreduced and washed as described above) were combined with a stock solution of glutaraldehyde $(1: 1 \mathrm{v} / \mathrm{v})$ to expose the cells to glutaraldehyde concentrations in the range from 0.02 to $0.08 \%(\mathrm{w} / \mathrm{v})$. The cells were incubated in glutaraldehyde for 10 minutes at room temperature $\left(22^{\circ} \mathrm{C}\right)$, and 
then the cross-linking reaction was quenched by adding about $50 \mathrm{~mL}$ of an isotonic glucose- and albumincontaining saline-phosphate (GASP) buffer $\left(1 \% \mathrm{w} / \mathrm{v}\right.$ bovine serum albumin, $9 \mathrm{mM} \mathrm{Na} 2 \mathrm{HPO}_{4}, 1.3 \mathrm{mM}$ $\mathrm{NaH}_{2} \mathrm{PO}_{4}, 140 \mathrm{mM} \mathrm{NaCl}, 5.5 \mathrm{mM}$ glucose, $\mathrm{pH} 7.4,290 \mathrm{mmol} \mathrm{kg}^{-1}$ ). Immediately after quenching, the treated RBCs were washed in the GASP buffer $(800 \times \mathrm{g}, 2 \mathrm{~min})$ and the excess buffer was removed to adjust the hematocrit to $40 \%$. In order to prepare RBC suspensions containing $0 \%, 10 \%, 50 \%$ and $100 \%$ of hardened cells, the $40 \%$ hematocrit suspension of RBCs that were exposed to $0.08 \%$ glutaraldehyde (as described above) was mixed a different ratios with a $40 \%$ hematocrit suspension of fresh, untreated RBCs.

\subsection{Diamide treatment}

A suspension of RBCs (prepared as described above) was washed once in GASP, and its hematocrit was adjusted to $45 \%$. A $1 \mathrm{~mL}$ aliquot of the $45 \%$ hematocrit suspension was treated with $125 \mu \mathrm{L}$ of a stock diamide solution (Sigma, St. Louis, USA) resulting in a $40 \%$ hematocrit suspension and a final concentration of diamide ranging from 0.02 to $0.08 \%$ (w/v). Samples were incubated for 20 minutes at $22^{\circ} \mathrm{C}$ and immediately analyzed. (Note that the final hematocrit of diamide-treated RBCs was $40 \%$, same as for the glutaraldehyde-treated RBCs).

\subsection{Ektacytometry measurements}

The treated RBC samples were prepared for analysis in the microfluidic ektacytometer RheoScan-D (RheoMeditech, Seoul, Korea) by re-suspending $6 \mu \mathrm{L}$ of the $40 \% \mathrm{RBC}$ suspension in $0.6 \mathrm{~mL}$ of PVP (polyvinylpyrrolidone) in accordance with the protocol suggested by the manufacturer. Each sample was analyzed using a $210 \mu \mathrm{m}$ height cartridge of the RheoScan-D to determine the elongation index (EI) for each RBC sample exposed to shear stresses ranging from 1 to $17 \mathrm{~Pa}$ (the limits of the instrument). EI values at $3 \mathrm{~Pa}$ and $17 \mathrm{~Pa}$ were recorded for diamide and glutaraldehyde concentrations ranging from $0.02 \%$ to $0.08 \%$ in $0.02 \%$ increments and then normalized with respect to the untreated sample. The normalized values $(n=4)$ were averaged and plotted versus their respective concentrations.

\subsection{Micro-pore filtration measurements}

The micro-pore filtration protocol used in this study has been previously described by us in detail [60]. Briefly, $5 \mu \mathrm{m}$ track-etched polycarbonate filters (Nuclepore ${ }^{\mathrm{TM}}$, Whatman, GE Healthcare Biosciences, Piscataway, NJ, USA) were pre-wetted with $40 \%$ ethyl alcohol and then incubated in GASP buffer for 1 hour before use. Filtration time (FT) was defined as the time it took a $200 \mu \mathrm{L}$ sample to pass through the filter. Each individual filter was calibrated using GASP buffer before performing the measurements on RBCs. The FT for treated RBCs was measured by timing the passage of $200 \mu \mathrm{L}$ of $1 \%$ hematocrit RBC suspensions being tested through the filter. The RBC filterability was calculated as the ratio of FT for GASP buffer (free of cells) and the FT for the RBC suspension being tested. These values were recorded for diamide and glutaraldehyde concentrations ranging from $0.02 \%$ to $0.08 \%$ in $0.02 \%$ increments and then normalized with respect to the untreated sample. The normalized values $(n=4)$ were averaged and plotted versus their respective concentrations. 


\subsection{Measurement of the artificial microvascular network perfusion}

The artificial microvascular network (AMVN) devices used in this study were fabricated using conventional photolithography techniques as described in detail in our previous studies [13, 23, 60]. Briefly, the pattern of AMVN was transferred onto a silicon wafer (University Wafer, South Boston, MA, USA) in bas-relief by exposing the wafer covered with a $5 \mu \mathrm{m}$ layer of negative photoresist (SU-8 2005, MicroChem Corp, Newton, MA, USA) to UV light through a chrome photomask (Photo Sciences Inc., Torrance, CA, USA). The three dimensional structure of the inlet and the outlet ports were created by depositing small droplets of SU-8 2007 photoresist onto the outlines of the inlet and the outlet, exposing these features to UV light and curing them [13,22]. This patterned silicon wafer acted as the master for molding many exact replicas of the AMVN out of polydimethylsiloxane (PDMS, Slygard 184, Dow Corning Corp., Midland, MI, USA). The access through-holes for the inlet $(4 \mathrm{~mm})$ and outlet $(2 \mathrm{~mm})$ of the PDMS replica were created using cylindrical biopsy punches (Acuderm Inc, Fort Lauderdale, FL, USA). The AMVN devices were assembled by exposing the patterned side of the PDMS replica and the surface of a glass slide (spin-coated with a thin film of PDMS) to air plasma (120 sec, PDC-3xG, Harrick Plasma, Ithaca, NY, USA) and sealing them together. Assembled AMVN devices were filled with $1 \%$ solution of mPEG-silane (Laysan Bio, Inc., Arab, AL, USA) in GASP solution, and were incubated at $22^{\circ} \mathrm{C}$ in $100 \%$ humidity overnight to suppress possible RBC adhesion to channel walls during the experiments.

The measurement of the AMVN perfusion was performed following the protocol developed in our previous studies $[13,60]$. Briefly, to perform an experiment, the assembled and treated AMVN devices were placed onto the mechanical stage of an inverted microscope (IX71, Olympus America Inc., Center Valley, PA, USA). The microscope was equipped with a high-speed, high-sensitivity camera (MC1362, Mikrotron GmbH, Unterschleissheim, Germany) connected to a Camera Link frame grabber (PIXCI E4, EPIX Inc., Buffalo Grove, IL, USA) installed in a desktop computer. A narrow band-pass blue filter (394 \pm 50 nm, B-390, Hoya Corp., Fremont, CA, USA) was used to improve the contrast of the images (RBCs appear dark in blue light). A simple water column was used to provide the pressure difference between the inlet and the outlet of the AMVN device. In the beginning of each AMVN measurement, the driving pressure difference was set to zero, the RBC sample was loaded into the inlets of the device, and the acquisition of short sequences of images (10 frames, every 10 seconds, for 10 minutes) was initiated. The driving pressure difference was then set to $20 \mathrm{~cm} \mathrm{H}_{2} \mathrm{O}$, and kept constant for the duration of the measurement. The acquired images were analyzed using customary algorithm written in MATLAB (The Math Works Inc., Natick, MA, USA) to determine the average RBC velocity in the arteriole of the AMVN. The steady state value of the average RBC velocity was used to calculate the overall flow rate through the arteriole (perfusion) of the AMVN [13]. The overall flow rate for each sample treated with glutaraldehyde or diamide (concentrations varying from $0.02 \%$ to $0.08 \%$, with $0.02 \%$ increments) were normalized with respect to the untreated sample. Normalized values were averaged over four individual trials and plotted versus their respective concentrations.

\subsection{Statistical analysis}

Data were presented as mean \pm standard deviation of individual measurements performed on multiple independent samples. The measurements of RBC deformability performed with different techniques were compared using the two-tailed, two-sample unequal variance $t$-test. The differences were deemed statistically significant for $p<0.05$. 


\section{Results}

\subsection{Conventional techniques for measuring RBC deformability}

Figure 1 illustrates the two conventional methodologies for measuring RBC deformability used in this study - micro-pore filtration (Fig. 1A) and ektacytometry (Fig. 1B). In the micro-pore filtration assay, the deformability of RBCs is evaluated by measuring the time it takes a set volume of a dilute RBC suspension to pass through a thin polycarbonate membrane containing capillary-size pores (usually a $5 \mu \mathrm{m}$ Nuclepore filter) [41, 60]. Unlike in the microvasculature in vivo, each RBC deforms only once while passing through a pore of the filter, which may limit its sensitivity. This measurement is known to be affected primarily by the size and sphericity of individual RBCs, and is often confounded by the plugging of pores by residual leukocytes and cellular clumps contaminating the tested sample, and / or by even a small fraction of non-deformable RBCs [42].

Ektacytometery evaluates RBC deformability by quantifying the degree of elongation of RBCs suspended in a highly-viscous solution of polyvinylpyrrolidone (PVP) and subjected to a well-defined shear stress field $[6,10,11,27]$. In this study, we used a microfluidic ektacytometer (RheoScan-D, Fig. 1B)
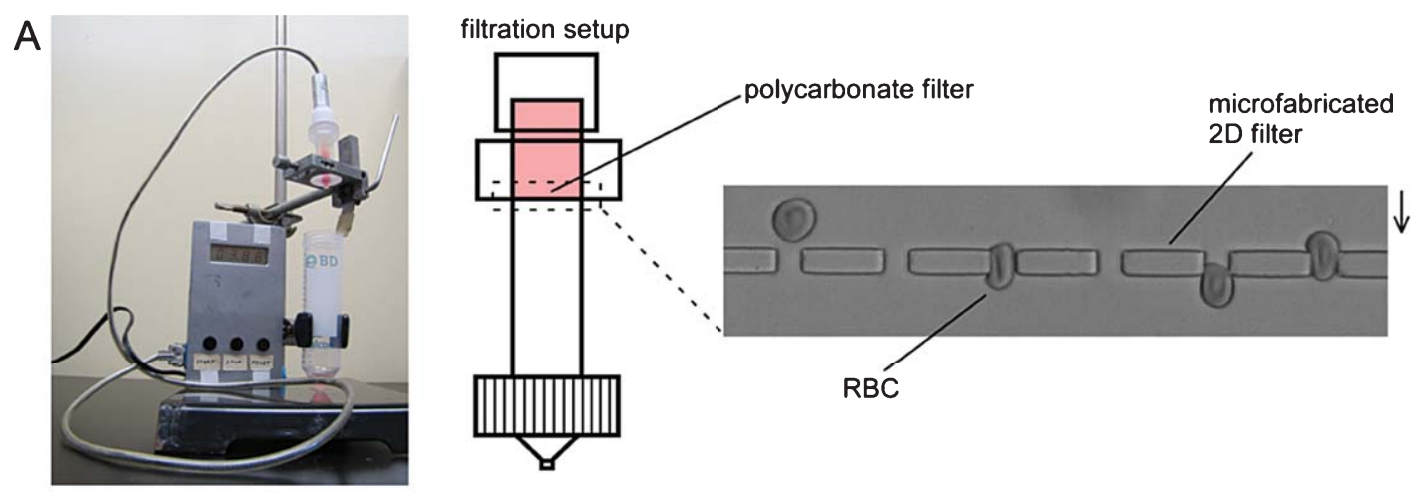

B
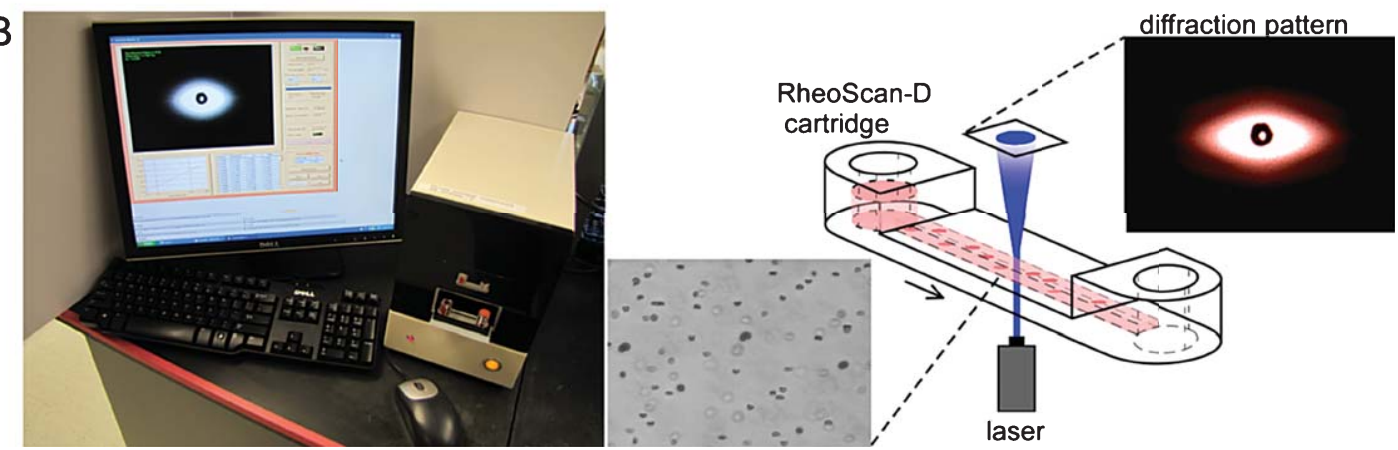

Fig. 1. Illustration of the two conventional technologies commonly used to evaluate RBC deformability. (A) In the micro-pore filtration technique, RBCs are passed through $5 \mu \mathrm{m}$ pores of a polycarbonate filter. The time it takes a pre-set volume of the RBC suspension to pass through the filter (filtration time) is measured to evaluate the ability of RBCs to undergo folding deformations when passing through narrow openings. (B) In an ektacytometer (RheoScan-D), a dilute suspension of RBCs in a highly viscous PVP solution is passed through a straight channel at various shear stresses. A laser beam is passed perpendicularly through the $\mathrm{RBC}$ suspension and the change in the diffraction pattern is quantified via the calculation of the elongation index to evaluate RBC deformability. 
to measure RBC deformability at shear stresses varying continually from $1 \mathrm{~Pa}$ up to $17 \mathrm{~Pa}$ (technical limit of the instrument) [61]. In the microfluidic ektacytometer, a laser beam is passed through a RBC sample flowing in a microfluidic channel $(0.2 \mathrm{~mm} \times 5 \mathrm{~mm} \times 40 \mathrm{~mm})$, and the change in the laser diffraction pattern is measured to quantify the RBC elongation, and therefore RBC deformability. Ektacytometry at low shear stresses (typically $3 \mathrm{~Pa}$ ) is most sensitive to RBC membrane properties, and at high shear stresses (e.g. $17 \mathrm{~Pa}$ ) it primarily reflects so-called "maximal deformability", which is determined by the RBC geometrical parameters (i.e., the cell surface area to volume ratio) [28, 46]. The measurement of RBC deformability using ektacytometry is confounded by several important factors, including: (a) the viscosity of the PVP solution is about 30-times larger than that of plasma, which is not physiological and thus completely changes the mechanical response of RBCs to shear (i.e. RBCs subjected to shear in plasma flip, not elongate), and (b) the hematocrit of RBC suspension tested is extremely low $(<0.5 \%)$ and thus ektacytometry cannot account for RBC deformations due to cellular collisions (which are abundant in real blood flow).

\subsection{Perfusion of the artificial microvascular network}

Figure 2 illustrates the artificial microvascular network (AMVN) device used in this study [13, 23]. This microfluidic device incorporated a network of capillary-size, $5 \mu \mathrm{m}$-deep microchannels ranging in width from 5 to $70 \mu \mathrm{m}$, arranged in a pattern inspired by the architecture of rat mesenteric microvasculature in vivo $[36,59,60]$. The fabrication of the AMVN device and the methodology of the AMVN perfusion measurement has been recently described in detail elsewhere [13]. To perform a measurement of the AMVN perfusion, we passed a $40 \%$ hematocrit suspension of RBCs through the network under a constant pressure difference between the inlet and the outlet $\left(\Delta P=20 \mathrm{cmH}_{2} \mathrm{O}\right)$, and quantified the overall flow rate through the venule of the network (Fig. 2A). The value of the AMVN perfusion rate reflects the effective ability of RBCs to undergo a wide range of deformations as the cells pass through the network of artificial capillaries ranging in size from $5 \mu \mathrm{m}$ to $70 \mu \mathrm{m}$ [13]. Figure 2 demonstrates the various deformations experienced by RBCs flowing at a $40 \%$ hematocrit through the AMVN. These include the most basic deformations of the cells into the characteristic bullet (Fig. 2D) and parachute (Fig. 2E) shapes, as well as deformations in larger vessels (Fig. 2G) and in bifurcations (Fig. 2C and F) of the network due to cell-cell and cell-wall interactions. The primary advantage of the AMVN device is that it directly measures the effective ability of RBCs to perfuse a microvascular network, and performs this highly integrative measurement under physiologically-relevant conditions, suspended in normal viscosity buffers or plasma at physiological hematocrits, or even as whole blood. Much like in the AMVN, RBCs in vivo must be able to deform in multiple ways to effectively perfuse the microvasculature and perform their primary function of delivering oxygen to tissues. In this study, we used the AMVN as a surrogate of the real microvasculature.

\subsection{Comparison of the conventional deformability measurements with the ability of RBCs to perfuse the AMVN}

Figure 3 demonstrates the results of the comparison between the measurements of RBC deformability performed using the two conventional techniques (micropore filtration and ektacytometry) and the ability of the same RBCs to perfuse the AMVN. To perform this comparison, we used three types of RBC samples with artificially altered overall deformability: (1) healthy, fresh RBCs exposed to graded concentrations of glutaraldehyde, (2) healthy, fresh RBCs exposed to graded concentrations of diamide, and (3) a mixture of healthy, fresh RBCs with RBCs rendered non-deformable by exposure to $0.08 \%$ glutaraldehyde. In all 

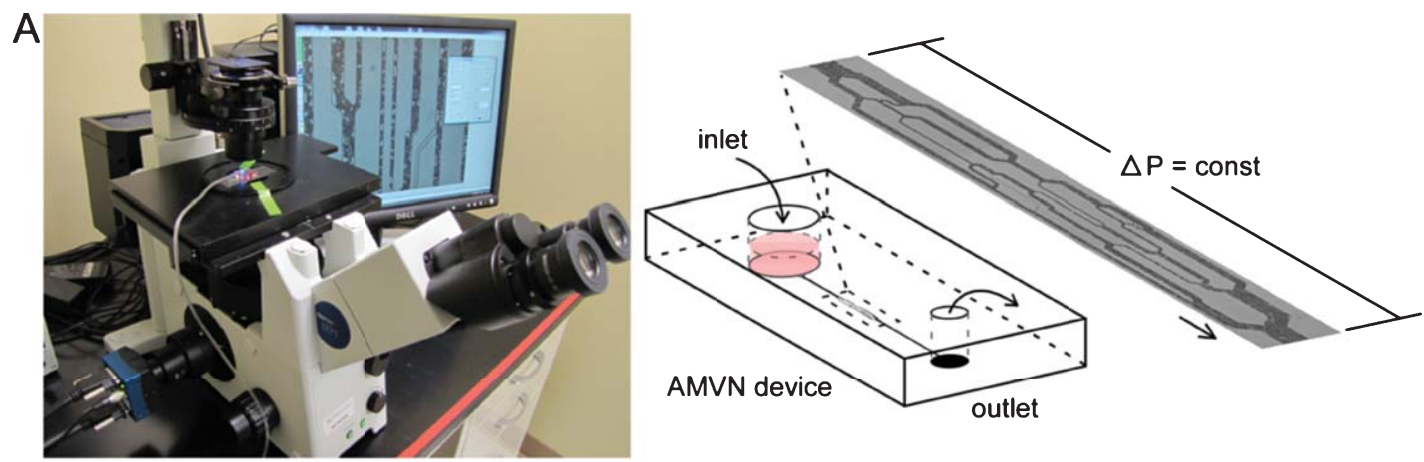

$\mathrm{B}$
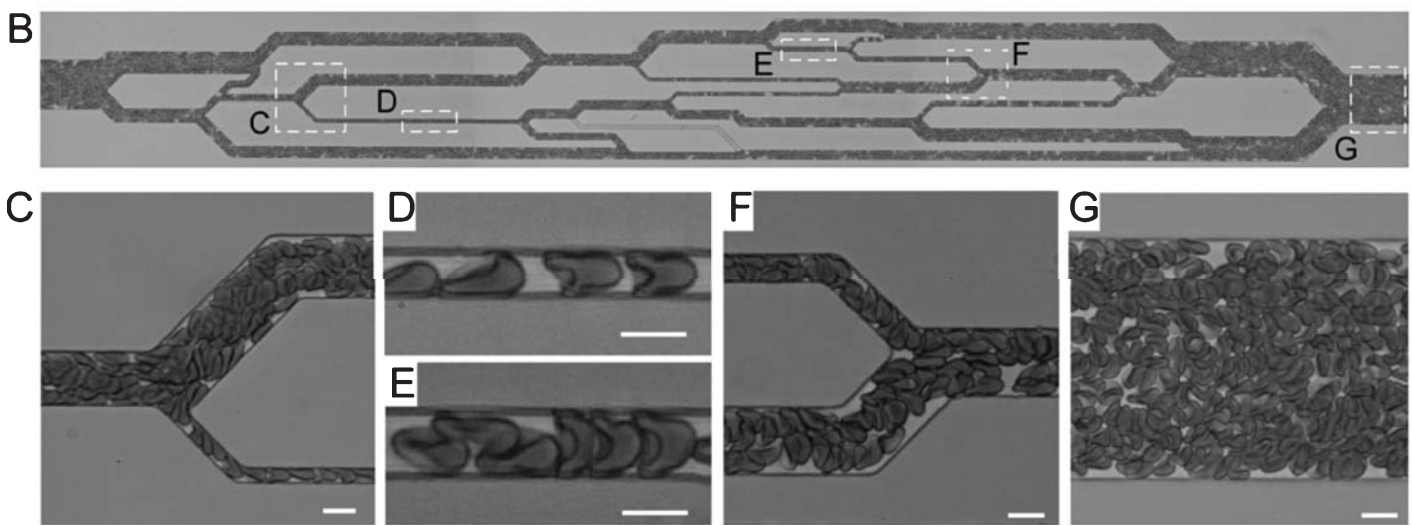

Fig. 2. Illustration of the artificial microvascular network (AMVN) device. (A) In the AMVN device, a $40 \%$ hematocrit suspension of RBCs is passed through a network of artificial capillaries under a constant pressure difference between the inlet and the outlet, and the overall flow rate in the venule of the AMVN is quantified. (B) The AMVN perfusion rate evaluates the effective ability of RBCs to undergo a variety of deformations as they pass through the network of artificial capillaries ranging in size from $5 \mu \mathrm{m}$ to $70 \mu \mathrm{m}$. (C) RBCs deform as a result of collisions with other cells and vessel walls at capillary bifurcations. (D) RBCs passing through the narrowest capillaries assume the characteristic bullet-like shape. (E) RBCs deform into the parachute shape in larger capillaries. (F) RBCs experience significant deformations due to the convergence of two streams moving with different velocities. (G) In largest vessels of the AMVN, RBCs undergo shear-induced deformations as well as deformations caused by multiple collisions with other cells in bulk flow. The flow direction is from left to right in all images. Scale bars are $10 \mu \mathrm{m}$.

samples, RBCs were re-suspended in GASP buffer rather than in autologous plasma to make sure that the AMVN perfusion measurements reflected RBCs mechanical properties rather than viscosity of the suspending medium or RBC aggregation behavior, and to maintain consistency with the previous work on ektacytometry and micro-pore filtration, most of which was done on RBCs re-suspended in various buffers rather than in autologous plasma. For each RBC sample, all there measurements (micropore filtration, ektacytometry and the AMVN perfusion) were performed simultaneously.

The measurement of RBC deformability using ektacytometry at both low (3 Pa) and high (17 Pa) shear stresses declined nearly linearly with the increasing concentration of glutaraldehyde (Fig. 3A). The value of RBC deformability measured using the micropore filtration technique stayed relatively insensitive to the lower range of glutaraldehyde concentrations (0-0.04\%), and declined precipitously for the concentrations of glutaraldehyde higher than $0.04 \%$ (Fig. 3A). The two techniques did not agree with each other, but for the highest concentration of glutaraldehyde, $0.08 \%$, at which point both techniques showed nearly complete lack of deformability for the treated RBCs. The ability of same RBCs to perfuse 

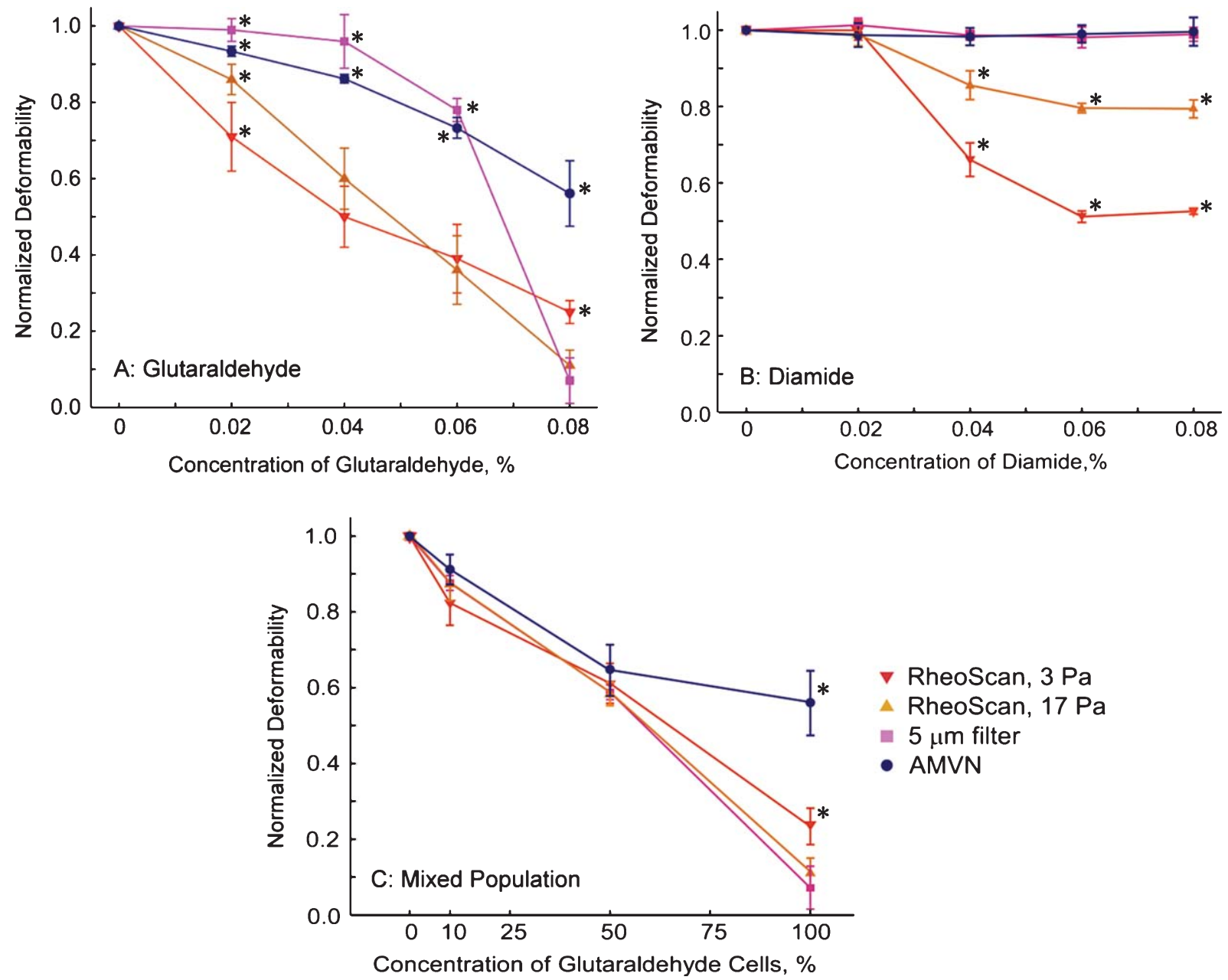

Fig. 3. A comparison of the normalized deformability indices measured using different methodologies for RBCs incubated with graded concentrations of (A) glutaraldehyde and (B) diamide, and (C) for reconstituted samples containing a mixture of healthy normal cells and varying percentage of non-deformable RBCs hardened by incubation with $0.08 \%$ glutaraldehyde. Each data point represents mean \pm standard deviation of $n=4$ samples ( ${ }^{*} p<0.05$ with respect to every other measurement technique).

the AMVN declined linearly with the increasing concentration of glutaraldehyde, to about $60 \%$ for the maximal concentration glutaraldehyde used in this study $(0.08 \%)$. Neither ektacytometry nor micropore filtration could accurately predict the AMVN perfusion, with ektacytometry significantly overestimating and micropore filtration underestimating (except for $0.08 \%$ ) the effect of the glutaraldehyde-induced impairment of RBC deformability on the network perfusion. For example, at the glutaraldehyde concentration of $0.04 \%$, ektacytometry showed a reduction in RBC deformability of $50 \%$ at low shear stress (3 $\mathrm{Pa}$ ) and $40 \%$ at high shear stress $(17 \mathrm{~Pa})$, whereas micropore filtration showed only a $4 \%$ reduction in RBC deformability and the AMVN perfusion was reduced by $14 \%$ for the same RBC sample (Fig. 3A).

Incubation of RBCs with increasing concentrations of diamide did not produce a measurable change in RBC deformability as measured by the micropore filtration technique, and did not affect the ability of same RBCs to perfuse the AMVN (Fig. 3B). In this sense, the micropore filtration and the AMVN 
perfusion measurements were in excellent agreement with each other. In sharp contrast, the measurement of deformability of the same RBCs performed using ektacytometry declined precipitously starting at $0.02 \%$ diamide concentration, reaching its minimal level plateau of $50 \%$ for low ( $3 \mathrm{~Pa}$ ) and $80 \%$ for high (17 Pa) shear stresses at concentrations higher than $0.06 \%$ (Fig. 3B). The ektacytometry measurements could not predict the ability of the same RBCs to perfuse the AMVN for any concentration of diamide above $0.02 \%$.

In addition to the samples in which the deformability of all RBCs was affected by the graded exposure to either glutaraldehyde or diamide, we also investigated how well ektacytometry and micropore filtration could predict the AMVN perfusion for samples comprising a mixture of deformable RBCs and nondeformable RBCs (Fig. 3C). To prepare these samples we mixed healthy, normal RBCs with varying proportions of RBCs hardened by the treatment with $0.08 \%$ glutaraldehyde, the maximal concentration of glutaraldehyde used in this study that resulted in nearly complete loss of deformability by treated RBCs as measured using ektacytometry and micropore filtration (Fig. 3A). The deformability of RBCs in the mixed samples declined linearly with the increasing fraction of non-deformable RBCs as measured using either micropore filtration or ektacytometry at high and low shear rates (Fig. 3C). Both ektacytometry and micropore filtration were able to predict the ability of the mixed samples to perfuse the AMVN very well, but only up to the $50: 50$ ratio of deformable and non-deformable RBCs. An increase in the fraction of nondeformable RBCs to $100 \%$ resulted in only about $8 \%$ reduction of AMVN perfusion, while conventional techniques showed a significant further decline of RBC deformability: 38\% for ektacytometry at low (3 Pa) shear rate, $47 \%$ for ektacytometry at high $(17 \mathrm{~Pa})$ shear rate, and $52 \%$ for micropore filtration (Fig. 3C).

\section{Discussion}

The deformability of RBCs is a multifactorial property that depends on membrane elasticity, cytoplasmatic viscosity, volume-to-surface ratio and the tank treading motion, with each of these variables affected differently by various pathophysiological processes and by the treatments used in this study [54, 58]. Our results suggest that neither ektacytometry nor micropore filtration can reliably predict the ability of RBCs with impaired deformability to perfuse a network of capillary microchannels (Fig. 3). Micropore filtration and ektacytometry evaluate two rather different aspects of RBC rheology - micropore filtration measures only the ability of RBCs to undergo folding deformations (relevant primarily to their entry into narrow capillaries), whereas ektacytometry measures only the ability of RBCs to elongate when subjected to a very well defined shear stress field in a highly viscous environment. The primary advantage of our approach over these two commonly used techniques is that in the AMVN RBCs experience a much wider spectrum of deformations, which includes the folding deformations in capillaries and the shear-induced deformations in larger vessels, and their properties are tested while suspended in normal-viscosity buffers (or even in blood plasma) at hematocrits that are physiologically-high and highly variable across vessels of different size (much like in real microvasculature [32, 33, 38, 70]) [13, 60]. In this sense, the measurement of AMVN perfusion rate provides a more realistic assessment of RBC rheology and (because the AMVN evaluates RBC mechanical properties directly in terms of microvascular perfusion) the results of this assessment should be easier to interpret clinically and may have more significant diagnostic implications [46].

One of the most important differences between the conventional techniques for measuring RBC deformability, and the AMVN perfusion measurement is the biomimetic ability of the AMVN to resist 
the detrimental effect of plugging of individual capillaries by non-deformable cells on the overall network perfusion. In many physiologically relevant conditions (e.g. sickle cell disease, malaria, blood transfusion), the distribution of deformability throughout the RBC population is not homogeneous, with only a fraction of all RBCs sustaining a significant impairment of their mechanical properties. In our experiments with samples containing mixtures of healthy, normal RBCs and RBCs rendered non-deformable by treatment with $0.08 \%$ glutaraldehyde (see Fig. 3A micropore filtration) [60] the AMVN perfusion for these samples declined almost linearly with the increasing fraction of non-deformable RBCs up to $50 \%$, in good agreement with the two conventional techniques (Fig. 3C). The subsequent increase of the fraction of non-deformable cells from $50 \%$ to $100 \%$ however, resulted in only an $8 \%$ reduction of the AMVN perfusion, whereas there was a further decline in RBC deformability of $52 \%$ as measured by micropore filtration, and a $38 \%$ decline for low shear (3 Pa) and a $47 \%$ decline for high shear (17 Pa) ektacytometry (Fig. 3C). Overall, the AMVN perfusion declined by less than $50 \%$ before reaching a plateau, suggesting that the flow of blood through the network was diverted to the larger microchannels, bypassing the plugged capillaries [60]. This behavior reflects a similar microcirculatory dynamics observed in vivo, where in the AMVN as in mammalian microcirculatory beds, RBCs have multiple pathways through which they can pass even if smaller vessels are occluded by less deformable RBCs $[41,72]$.

The main objective of this study was to investigate whether the measurements of RBC deformability performed using the two conventional techniques (ektacytometry and micro-pore filtration) could predict the ability of same RBCs to perfuse an artificial microvascular network. In this context, we used the AMVN with a particular architecture (Fig. 2B) as a representative example of such a network. A significant alteration of the network architecture would affect the specifics of the blood flow dynamics within the network and therefore the measurement of the network perfusion. This feature is in fact an important advantage of our approach because the architecture of the AMVN could, in principle, be designed to mimic the microvasculature of a target organ of interest (e.g. kidney, brain, heart) and thus enable an organ-specific prediction of the impact of $\mathrm{RBC}$ mechanical properties on microvascular perfusion. We believe, however, the results of our current study and conclusions we drew from them in this paper are general in nature and therefore will hold true with respect to a reasonable variation of the network architecture. A separate future study designed to measure the effect of systematic variations in the network architecture on the perfusion measurement would be able to test this hypothesis definitively.

Glutaraldehyde is a non-specific protein cross-linker that affects proteins present in all three structural regions of a mature $\mathrm{RBC}$, including those in the cytosol, as well as the cytoskeletal and transmembrane proteins $[7,15,24,26,44,48,68]$. Diamide is a sulfhydryl-oxidizing reagent that exclusively cross-links spectrin via the formation of disulfide bonds within the RBC cytoskeleton [5, 24]. Controlled exposure to glutaraldehyde and/or diamide is widely used in the literature to mimic the pathophysiological impairment of RBC mechanical properties occurring naturally, particularly in validation and sensitivity studies of new $\mathrm{RBC}$ deformability measurement techniques $[4,5,24,28,41,60,61]$. A high sensitivity of a technique for measuring RBC deformability to either of these chemical treatments, however, may not necessarily mean a similarly high sensitivity to the other treatment (Fig. 3A-B).

In the ektacytometer, the deformability of RBCs is measured by quantifying the diffraction pattern of a laser beam passing through a $\sim 0.5 \%$ hematocrit suspension of RBCs in a high-viscosity $(30 \mathrm{mPa} \cdot \mathrm{s})$ solution of poly(vinylpyrrolidone). When subjected to shear stress in such a highly viscous environment ( $>10 \times$ viscosity of plasma), RBCs do not flip, but rather elongate in response, affecting the laser diffraction pattern and the readout of the technique. Our measurements showed that RBC elongation under the conditions of ektacytometry is highly sensitive to both the treatment of RBCs with glutaraldehyde 
(Fig. 3A) and with diamide (Fig. 3B). This was not however the case for either micropore filtration or the AMVN perfusion measurements.

In micropore filtration, the deformability of $\mathrm{RBCs}$ is measured by quantifying the time it takes a fixed volume of a dilute suspension of RBCs (in saline or another normal viscosity aqueous buffer solution) to pass through $5-\mu \mathrm{m}$ pores of a thin membrane. To pass through the smaller pores, each RBC in the suspension must undergo a single folding deformation. Under the conditions of the micropore filtration assay, the ability of RBCs to fold into the capillary-size pores declined mildly after the treatment with the lowest concentrations of glutaraldehyde. At the concentrations higher than $0.04 \%$, the RBC mechanical properties deteriorated precipitously, losing nearly all of their original deformability at the maximal exposure (Fig. 3A) (in complete agreement with previous studies) [41, 60]. In a striking contrast with ektacytometry and despite the evident sensitivity to glutaraldehyde, the ability of treated RBC to negotiate the capillary-size pores in the micropore filtration assay was completely unaffected by the treatment with diamide (Fig. 3B). Similarly, the ability of RBCs to perfuse the AMVN declined linearly with increasing concentration of glutaraldehyde (Fig. 3A), but remained completely unaffected by exposure of RBCs to diamide over the entire range of concentrations (Fig. 3B). A possible explanation of our observations is that spectrin-specific cross-linking of the cytoskeleton affects the ability of RBCs to elongate in response to applied shear stress when suspended in a high-viscosity buffer (ektacytometry), but does not affect the ability of RBCs to deform when passing through capillary-size pores (micropore filtration) and traversing a network of capillary microchannels (AMVN). Our findings are consistent with previous studies, also reporting differential sensitivity of various in vitro measurement techniques (including ektacytometry and micropore filtration) to the treatments with glutaraldehyde and diamide [5, 24, 49].

By the same token, it is highly unlikely that treating normal, healthy RBCs with either glutaraldehyde or diamide represents any of the changes in RBC mechanical properties occurring naturally as a functional manifestation of genetic abnormalities, due to metabolic alterations, in response to a changing biochemical microenvironment or as a result of a pathological process. In this context, high sensitivity of a particular measurement technique to the chemically induced impairment of RBC deformability does not necessarily mean a similar level of sensitivity to the real changes occurring in vivo. For example, Berezina et al. used the micropore filtration technique to quantify the progressive deterioration of $\mathrm{RBC}$ mechanical properties during a 6-week hypothermic storage, and found that by day 42 of storage RBC deformability was $46 \%$ lower than for fresh cells [9]. In a more recent study, Henkelman et al. used ektacytometry to investigate the evolution of RBC mechanical properties during 7 weeks of hypothermic storage. They found that deformability of stored RBCs did not change at the shear stress of 3.9 Pa throughout storage. At a higher shear stress of $50 \mathrm{~Pa}$, the deformability of stored RBCs in the beginning of storage ( 3 days after donation) was about $9 \%$ higher than for fresh RBCs, but then gradually decreased to about $2 \%$ higher than for fresh RBCs by the end of storage [30]. For comparison, in our own recent study we measured a $26 \pm 4 \%$ decline in the ability of RBCs to perfuse the AMVN after a 41-day hypothermic storage [13].

In our measurements performed on the same RBC samples simultaneously, micropore filtration was significantly less sensitive to the impairment of RBC deformability caused by the treatment with glutaraldehyde than ektacytometry (Fig. 3A); unlike ektacytometry, micropore filtration was also completely insensitive to the treatment with diamide (Fig. 3B). Using these data it is logical to make a conclusion that ektacytometry is a more sensitive technique for measuring RBC deformability than micropore filtration. Yet, when used to quantify the deterioration of RBC mechanical properties caused by the same biological process (storage lesion), micropore filtration reported a significant loss of nearly half of RBC deformability by the end of storage [9], while the ektacytometry measurements suggested a slight improvement with respect to fresh RBCs [30]. The marked disagreement between the sensitivity of the two techniques to the 
chemically-induced and the natural impairment of RBC mechanical properties highlights the danger of using the treatment with glutaraldehyde and/or diamide as the benchmark when developing, optimizing and validating novel techniques for measuring RBC deformability. More studies focused on the side-byside comparison of various RBC deformability measurement techniques, and more representative models of the natural RBC deformability impairment for calibrating these techniques in vitro are urgently needed.

\section{Conclusion}

This study compared the measurements of RBC deformability performed using two very commonly employed techniques, micropore filtration and ektacytometry, and investigated how well these measurements could predict the ability of same RBCs to perfuse an artificial microvascular network (AMVN). The two conventional techniques did not agree with each other, and could not accurately predict the ability of the RBCs with artificially impaired deformability to perfuse the AMVN. Our study reinforces the notion that RBC deformability is not a unique property, but rather a property that is operationally defined by the measurement methodology. This seemingly obvious realization highlights the need for any study of RBC deformability to employ multiple measurement methods in order to benefit from the descriptive strengths of the different technologies and perform a multi-parameter, comprehensive evaluation of RBC mechanical response to deforming forces. A vast majority of RBC deformability studies published to date base their conclusions on a single measurement method. While the insights gleaned from these studies are certainly valuable, they may give a one-dimensional, biased assessment because of the attempt to reduce the multifaceted nature of such a complex phenomenon as RBC deformability to a single numerical value. One way towards a much more balanced and potentially more clinically meaningful assessment of RBC mechanical properties is to invest a significant research effort into the development and careful validation of novel biomimetic techniques and devices that can test RBC behavior in the mechanical microenvironment closely mimicking the dynamics of blood flow in the microvasculature. We speculate that the measurement of perfusion of an artificial microvascular network (AMVN) to evaluate the mechanical properties of RBCs is a significant step towards this important goal.

\section{Acknowledgments}

This work was supported in part by an award from the National Blood Foundation (SSS), a 2012 NIH Directors' Transformative Research Award (1R01HL117329-01, PI: SSS), a grant from the National Science Foundation and the Louisiana Board of Regents through the Louis Stokes-Louisiana Alliance for Minority Participation at Tulane (JMS), and a grant from the Newcomb Tulane College Honors Program (TGC).

\section{References}

[1] K. Araki and J.M. Rifkind, The rate of osmotic hemolysis: A relationship with membrane bilayer fluidity, Biochim Biophys Acta 645(1) (1981), 81-90.

[2] D.L. Bader and M.M. Knight, Biomechanical analysis of structural deformation in living cells, Med Biol Eng Comput 46(10) (2008), 951-963.

[3] R. Banerjee, K. Nageshwari and R.R. Puniyani, The diagnostic relevance of red cell rigidity, Clin Hemorheol Microcirc 19(1) (1998), 21-24. 
[4] O.K. Baskurt, M.R. Hardeman, M. Uyuklu, P. Ulker, M. Cengiz, N. Nemeth, S. Shin, T. Alexy and H.J. Meiselman, Comparison of three commercially available ektacytometers with different shearing geometries, Biorheology 46(3) (2009), 251-264.

[5] O.K. Baskurt and F. Mat, Importance of measurement temperature in detecting the alterations of red blood cell aggregation and deformability studied by ektacytometry: A study on experimental sepsis in rats, Clin Hemorheol Microcirc 23(1) (2000), 43-49.

[6] R. Bayer, S. Caglayan, R. Hofmann, and D. Ostuni. Laser diffraction of RBC: The method and its pitfalls. SPIE. 1994.

[7] P.S. Becker, C.M. Cohen and S.E. Lux, The effect of mild diamide oxidation on the structure and function of human erythrocyte spectrin, J Biol Chem 261(10) (1986), 4620-4628.

[8] E. Bennett-Guerrero, T.H. Veldman, A. Doctor, M.J. Telen, T.L. Ortel, T.S. Reid, M.A. Mulherin, H. Zhu, R.D. Buck, R.M. Califf and T.J. McMahon, Evolution of adverse changes in stored RBCs, Proc Natl Acad Sci U S A 104(43) (2007), $17063-17068$.

[9] T.L. Berezina, S.B. Zaets, C. Morgan, C.R. Spillert, M. Kamiyama, Z. Spolarics, E.A. Deitch and G.W. Machiedo, Influence of storage on red blood cell rheological properties, J Surg Res 102(1) (2002), 6-12.

[10] M. Bessis and N. Mohandas, A diffractometric method for the measurement of cellular deformability, Blood cells 1(3) (1975), 307-313.

[11] M. Bessis, N. Mohandas and C. Feo, Automated ektacytometry: A new method of measuring red cell deformability and red cell indices, Blood Cells 6(3) (1980), 315-327.

[12] C.D. Brown, H.S. Ghali, Z. Zhao, L.L. Thomas and E.A. Friedman, Association of reduced red blood cell deformability and diabetic nephropathy, Kidney Int 67(1) (2005), 295-300.

[13] J.M. Burns, X. Yang, O. Forouzan, J.M. Sosa and S.S. Shevkoplyas, Artificial microvascular network: A new tool for measuring rheologic properties of stored red blood cells, Transfusion 52(5) (2012), 1010-1023.

[14] R.T. Card, N. Mohandas and P.L. Mollison, Relationship of post-transfusion viability to deformability of stored red cells, Br J Haematol 53(2) (1983), 237-240.

[15] J.A. Chasis and S.L. Schrier, Membrane deformability and the capacity for shape change in the erythrocyte, Blood 74(7) (1989), 2562-2568.

[16] S. Chien, Red cell deformability and its relevance to blood flow, Annu Rev Physiol 49 (1987), 177-192.

[17] L.G. Cima, D.E. Discher, J. Tong and M.C. Williams, A hydrodynamic interpretation of crisis in sickle cell anemia, Microvasc Res 47(1) (1994), 41-54.

[18] R. de Vroege, W.R. Wildevuur, J.A. Muradin, D. Graves and W. van Oeveren, Washing of stored red blood cells by an autotransfusion device before transfusion, Vox Sang 92(2) (2007), 130-135.

[19] A.M. Dondorp, P.A. Kager, J. Vreeken and N.J. White, Abnormal blood flow and red blood cell deformability in severe malaria, Parasitol Today 16(6) (2000), 228-232.

[20] A.M. Dondorp, F. Omodeo-Sale, K. Chotivanich, D. Taramelli and N.J. White, Oxidative stress and rheology in severe malaria, Redox Rep 8(5) (2003), 292-294.

[21] T.C. Fisher, R.B. Wenby and H.J. Meiselman, Pulse shape analysis of RBC micropore flow via new software for the cell transit analyser (CTA), Biorheology 29(2-3) (1992), 185-201.

[22] O. Forouzan, J.M. Burns, J.L. Robichaux, W.L. Murfee and S.S. Shevkoplyas, Passive recruitment of circulating leukocytes into capillary sprouts from existing capillaries in a microfluidic system, Lab on a Chip 11(11) (2011), 1924-1932.

[23] O. Forouzan, X. Yang, J.M. Sosa, J.M. Burns and S.S. Shevkoplyas, Spontaneous oscillations of capillary blood flow in artificial microvascular networks, Microvasc Res 84(2) (2012), 123-132.

[24] A.M. Forsyth, J. Wan, W.D. Ristenpart and H.A. Stone, The dynamic behavior of chemically "stiffened" red blood cells in microchannel flows, Microvasc Res 80(1) (2010), 37-43.

[25] M.H. Friedlander, R. Simon and G.W. Machiedo, The relationship of packed cell transfusion to red blood cell deformability in systemic inflammatory response syndrome patients, Shock 9(2) (1998), 84-88.

[26] L.W. Fung, B.O. Kalaw, R.M. Hatfield and M.N. Dias, Erythrocyte spectrin maintains its segmental motions on oxidation: A spin-label EPR study, Biophys J 70(2) (1996), 841-851.

[27] W. Groner, N. Mohandas and M. Bessis, New optical technique for measuring erythrocyte deformability with the ektacytometer, Clin Chem 26(10) (1980), 1435-1442.

[28] M. Hardeman, P. Goedhart, J. Dobbe and K. Lettinga, Laser-assisted optical rotational cell analyser (LORCA). I: A new instrument for measurement of various structural hemorheological parameters, Clinical Hemorheology 14(4) (1994), 605-619. 
[29] M. Hardeman, P. Goedhart and N. Schut, Laser-assisted optical rotational cell analyser (LORCA). II: Red blood cell deformability: Elongation index versus cell transit time, Clinical Hemorheology 14(4) (1994), 619-630.

[30] S. Henkelman, M.J. Dijkstra-Tiekstra, J. de Wildt-Eggen, R. Graaff, G. Rakhorst and W. van Oeveren, Is red blood cell rheology preserved during routine blood bank storage? Transfusion 50(4) (2010), 941-948.

[31] P. Izzo, A. Spagnuolo and A. Manicone, Assessment of erythrocyte deformability with the laser-assisted optical rotational cell analyzer (LORCA), Boll Soc Ital Biol Sper 75(1-2) (1999), 9-15.

[32] P.C. Johnson, D.L. Hudnall and J.H. Dial, Measurement of capillary hematocrit by photometric techniques, Microvascular Research 5(3) (1973), 351-356.

[33] F. Jung, J. Koscielny, C. Mrowietz, S. Wolf, H. Kiesewetter and E. Wenzel, Effect of hemodilution on systemic and capillary hematocrit, Infusionstherapie 17(5) (1990), 268.

[34] F. Jung, C. Mrowietz, B. Hiebl, R. Franke, G. Pindur and R. Sternitzky, Influence of rheological parameters on the velocity of erythrocytes passing nailfold capillaries in humans, Clin Hemorheol Microcirc 48(1) (2011), 129-139.

[35] A. Kearney-Schwartz, J.M. Virion, J.F. Stoltz, P. Drouin and F. Zannad, Haemorheological disturbances in hypertensive type 2 diabetic patients-influence of antihypertensive therapy, Fundam Clin Pharmacol 21(4) (2007), 387-396.

[36] M.F. Kiani, A.R. Pries, L.L. Hsu, I.H. Sarelius and G.R. Cokelet, Fluctuations in microvascular blood flow parameters caused by hemodynamic mechanisms, American Journal of Physiology-Heart and Circulatory Physiology 266(5) (1994), H1822-H1828.

[37] Y.K. Kim, E.H. Kwon, D.H. Kim, D.I. Won, S. Shin and J.S. Suh, Susceptibility of oxidative stress on red blood cells exposed to gamma rays: Hemorheological evaluation, Clin Hemorheol Microcirc 40(4) (2008), 315-324.

[38] B. Klitzman and B.R. Duling, Microvascular hematocrit and red cell flow in resting and contracting striated muscle, American Journal of Physiology-Heart and Circulatory Physiology 237(4) (1979), H481-H490.

[39] D. Koutsouris, R. Guillet, J.C. Lelievre, M.T. Guillemin, P. Bertholom, Y. Beuzard and M. Boynard, Determination of erythrocyte transit times through micropores. I-Basic operational principles, Biorheology 25(5) (1988), 763-772.

[40] D. Koutsouris, R. Guillet, R.B. Wenby and H.J. Meiselman, Determination of erythrocyte transit times through micropores. II- Influence of experimental and physicochemical factors, Biorheology 25(5) (1988), 773-790.

[41] H.H. Lipowsky, L.E. Cram, W. Justice and M.J. Eppihimer, Effect of erythrocyte deformability on in vivo red cell transit time and hematocrit and their correlation with in vitro filterability, Microvasc Res 46(1) (1993), 43-64.

[42] I. Lisovskaya, E. Shurkhina, V. Nesterenko, J. Rozenberg and F. Ataullakhanov, Determination of the content of nonfilterable cells in erythrocyte suspensions as a function of the medium osmolality, Biorheology 35(2) (1998), 141-154.

[43] H. Minamitani, K. Tsukada, E. Sekizuka and C. Oshio, Optical bioimaging: From living tissue to a single molecule: Imaging and functional analysis of blood flow in organic microcirculation, J Pharmacol Sci 93(3) (2003), 227-233.

[44] F.M. Morel, R.F. Baker and H. Wayland, Quantitation of human red blood cell fixation by glutaraldehyde, J Cell Biol 48(1) (1971), 91-100.

[45] A.G. Moutzouri, G.A. Athanassiou, D. Dimitropoulou, A.T. Skoutelis and C.A. Gogos, Severe sepsis and diabetes mellitus have additive effects on red blood cell deformability, J Infect 57(2) (2008), 147-151.

[46] M. Musielak, Red blood cell-deformability measurement: Review of techniques, Clin Hemorheol Microcirc 42(1) (2009), 47-64.

[47] N. Nemeth, I. Furka and I. Miko, Hemorheological changes in ischemia-reperfusion: An overview on our experimental surgical data, Clin Hemorheol Microcirc (2013). DOI: 10.3233/CH-131648

[48] S. Noji, S. Taniguchi and H. Kon, An EPR study on erythrocyte deformability, Prog Biophys Mol Biol 55(2) (1991), 85-105.

[49] E. Ogura, P.J. Abatti and T. Moriizumi, Measurement of human red blood cell deformability using a single micropore on a thin Si/sub 3/N/sub 4/film. Biomedical Engineering, IEEE Transactions on 38(8) (1991), 721-726.

[50] G.A.M. Pop, D.J. Duncker, M. Gardien, P. Vranckx, S. Versluis, D. Hasan and C.J. Slager, The clinical significance of whole blood viscosity in (cardio)vascular medicine, Neth Heart J 10(12) (2002), 512-516.

[51] N.J. Raat, A.J. Verhoeven, E.G. Mik, C.W. Gouwerok, R. Verhaar, P.T. Goedhart, D. de Korte, and C. Ince, The effect of storage time of human red cells on intestinal microcirculatory oxygenation in a rat isovolemic exchange model, Crit Care Med 33(1) (2005), 39-45; discussion 238-9.

[52] G. Reggiori, G. Occhipinti, A. De Gasperi, J.L. Vincent and M. Piagnerelli, Early alterations of red blood cell rheology in critically ill patients*, Critical Care Medicine 37(12) (2009), 3041.

[53] H.L. Reid, A.J. Barnes, P.J. Lock, J.A. Dormandy and T.L. Dormandy, A simple method for measuring erythrocyte deformability, J Clin Pathol 29(9) (1976), 855-858. 
[54] W.H. Reinhart and S. Chien, Roles of cell geometry and cellular viscosity in red cell passage through narrow pores, American Journal of Physiology-Cell Physiology 248(5) (1985), C473-C479.

[55] W.H. Reinhart, S.J. Danoff, S. Usami and S. Chien, Rheologic measurements on small samples with a new capillary viscometer, J Lab Clin Med 104(6) (1984), 921-931.

[56] W.H. Reinhart, C. Huang, M. Vayo, G. Norwich, S. Chien and R. Skalak, Folding of red blood cells in capillaries and narrow pores, Biorheology 28(6) (1991), 537-549.

[57] B. Riquelme, P. Foresto, M. D’Arrigo, F. Filippini and J. Valverde, Laser diffractometry technique: Clinical applications to vascular pathologies, Clin Hemorheol Microcirc 35(1-2) (2006), 277-281.

[58] H. Schmid-Schönbein and P. Gaehtgens, What is red cell deformability? Scandinavian Journal of Clinical \& Laboratory Investigation 41(S156) (1981), 13-26.

[59] S.S. Shevkoplyas, S.C. Gifford, T. Yoshida and M.W. Bitensky, Prototype of an in vitro model of the microcirculation, Microvascular Research 65(2) (2003), 132-136.

[60] S.S. Shevkoplyas, T. Yoshida, S.C. Gifford and M.W. Bitensky, Direct measurement of the impact of impaired erythrocyte deformability on microvascular network perfusion in a microfluidic device, Lab Chip 6(7) (2006), 914-920.

[61] S. Shin, J.X. Hou, J.S. Suh and M. Singh, Validation and application of a microfluidic ektacytometer (RheoScan-D) in measuring erythrocyte deformability, Clin Hemorheol Microcirc 37(4) (2007), 319-328.

[62] M.J. Simmonds, S. Sabapathy, G.C. Gass, S.M. Marshall-Gradisnik, L.J. Haseler, R.M. Christy and C.L. Minahan, Heart rate variability is related to impaired haemorheology in older women with type 2 diabetes, Clin Hemorheol Microcirc 46(1) (2010), 57-68.

[63] M. Singh and S. Shin, Changes in erythrocyte aggregation and deformability in diabetes mellitus: A brief review, Indian $J$ Exp Biol 47(1) (2009), 7-15.

[64] A. Stadler and O. Linderkamp, Flow behavior of neonatal and adult erythrocytes in narrow capillaries, Microvasc Res 37(3) (1989), 267-279.

[65] J. Stuart, Erythrocyte rheology, J Clin Pathol 38(9) (1985), 965-977.

[66] J. Stuart and G.B. Nash, Technological advances in blood rheology, Critical Reviews in Clinical Laboratory Sciences 28(1) (1990), 61-93.

[67] S. Svenmarker, E. Jansson, H. Stenlund and K.G. Engstrom, Red blood cell trauma during cardiopulmonary bypass: Narrow pore filterability versus free haemoglobin, Perfusion 15(1) (2000), 33-40.

[68] A. Szwarocka, A. Kowalczyk, D. Lubgan and Z. Jozwiak, The combined effect of IDA and glutaraldehyde on the properties of human erythrocytes, Int J Pharm 220(1-2) (2001), 43-51.

[69] I.A. Tikhomirova, A.O. Oslyakova and S.G. Mikhailova, Microcirculation and blood rheology in patients with cerebrovascular disorders, Clin Hemorheol Microcirc 49(1-4) (2011), 295-305.

[70] R. Yen and Y. Fung, Inversion of Fahraeus effect and effect of mainstream flow on capillary hematocrit, Journal of Applied Physiology 42(4) (1977), 578-586.

[71] L. Zehnder, T. Schulzki, J.S. Goede, J. Hayes and W.H. Reinhart, Erythrocyte storage in hypertonic (SAGM) or isotonic (PAGGSM) conservation medium: Influence on cell properties, Vox Sang 95(4) (2008), 280-287.

[72] B.W. Zweifach, Functional behavior of the microcirculation, American Lecture Series, 1961, Charles C. Thomas, Publisher Springfield, Illinois, USA. 